\title{
Guaranteed Stability with Subspace Methods
}

\author{
J.M.Maciejowski \\ Cambridge University Engineering Dept. \\ Cambridge, CB2 1PZ, England. \\ Email: jmm@eng.cam.ac.uk, Fax: +44 1223332662 \\ 19 July 1994 \\ Revised 10 February 1995 \\ Submitted to Systems and Control Letters, Paper \#2694.
}

\begin{abstract}
We show how stability of models can be guaranteed when using the class of identification algorithms which have become known as 'subspace methods'. In many of these methods the ' $A$ ' matrix is obtained (or can be obtained) as the product of a shifted matrix with a pseudo-inverse. We show that whenever the shifted matrix is formed by introducing one block of zeros in the appropriate position, then a stable model results. The cost of this is some (possibly large) distortion of the results, but in some applications that is outweighed by the advantage of guaranteed stability.
\end{abstract}

Keywords: Subspace methods, Identification, Stability, Multivariable systems, State-space models, Discrete-time.

\section{Introduction}

So-called 'subspace methods' have proved extremely successful at black-box identification of multivariable linear state-space models from data, and are consequently of great current interest $[6,7,8,10,11,13]$. In this paper we show that stability of the models obtained can be guaranteed very simply and in a uniform manner for many of the published variations of subspace methods.

Our use of the phrase 'subspace method' is wider than its use by some authors. We include in it all state-space identification methods which are based more or less explicitly on realization theory of linear systems. In particular, we include those methods which begin by forming Hankel matrices from impulse response or covariance estimates, such as $[5,6,16]$, and methods based on Hankel matrices built up from input and output data [7, 8, 11, 12, 13]. This latter class of methods apparently has its origins in $[4,1]$; an important feature of this class, and one which is regarded by some as the distinguishing characteristic of 'subspace methods', is that a state sequence is estimated from the input-output data before the system matrices are estimated. This idea seems to have been introduced in $[7,15]$.

All subspace methods yield discrete-time state-space models such as

$$
\begin{aligned}
x(t+1) & =A x(t)+B u(t) \\
y(t) & =C x(t)+D u(t)
\end{aligned}
$$


(where $x(t) \in \mathbf{R}^{n}, u(t) \in \mathbf{R}^{m}, y(t) \in \mathbf{R}^{p}$ ); many of them incorporate extensions to models with both measured and unmeasured inputs, stochastic models, etc, but we do not need the details here. These algorithms frequently estimate the ' $A$ ' matrix by exploiting some kind of 'shift-invariance' property. In $[6,16]$ this is the shift-invariance of the extended observability matrix

$$
\Omega=\left[\begin{array}{c}
C \\
C A \\
C A^{2} \\
\vdots
\end{array}\right]
$$

or of the extended controllability matrix

$$
\Gamma=\left[B, A B, A^{2} B, \ldots\right] .
$$

'Shift-invariance' here means that

$$
\Omega A=\Omega^{\uparrow}
$$

and

$$
A \Gamma=\Gamma^{\leftarrow}
$$

where

$$
\Omega^{\uparrow}=\left[\begin{array}{c}
C A \\
C A^{2} \\
C A^{3} \\
\vdots
\end{array}\right]
$$

and

$$
\Gamma^{\leftarrow}=\left[A B, A^{2} B, A^{3} B, \ldots\right] .
$$

Many subspace algorithms form a finite estimate $\hat{\Omega}$ of $\Omega$ and obtain an estimate $\hat{A}$ of $A$ as

$$
\hat{A}=\hat{\Omega}^{\dagger} \hat{\Omega}^{\uparrow}
$$

where $(.)^{\dagger}$ denotes the Moore-Penrose pseudo-inverse. In Kung's algorithm [6] an estimate $\hat{\Gamma}$ of $\Gamma$ is also available, and it is possible to use

$$
\hat{A}=\hat{\Gamma}^{\leftarrow} \hat{\Gamma}^{\dagger}
$$

In some subspace algorithms a similar, but less complete, shift-invariance is used. Let $X_{t}=$ $[x(t), x(t+1), \ldots]$, and let $U_{t}$ and $Y_{t}$ be defined similarly. Then

$$
\left[\begin{array}{c}
X_{t}^{\leftarrow} \\
Y_{t}
\end{array}\right]=\left[\begin{array}{cc}
A & B \\
C & D
\end{array}\right]\left[\begin{array}{c}
X_{t} \\
U_{t}
\end{array}\right]
$$

where $X_{t}^{\leftarrow}=X_{t+1}$. In [7, 8, 11] and the 'elementary MOESP' algorithm of [13] an estimate $\hat{X}_{t}$ is first obtained, and the model is estimated from

$$
\left[\begin{array}{ll}
\hat{A} & \hat{B} \\
\hat{C} & \hat{D}
\end{array}\right]=\left[\begin{array}{c}
\hat{X}_{t}^{\leftarrow} \\
Y_{t}
\end{array}\right]\left[\begin{array}{c}
\hat{X}_{t} \\
U_{t}
\end{array}\right]^{\dagger}
$$


In this case the method we propose does not work, because $\hat{A} \neq \hat{X}_{t}^{\leftarrow} \hat{X}_{t}^{\dagger}$, but alternative solution strategies are available, with which our method can be applied. Typically these involve 'backing off' to estimate $A$ and $C$ from an estimate of the extended observability matrix, as in equation (9) above [12]. The 'ordinary MOESP' algorithm of [13] uses such a strategy. In the 'purely stochastic' case, when there are no measured inputs, equation (12) reduces to

$$
\left[\begin{array}{l}
\hat{A} \\
\hat{C}
\end{array}\right]=\left[\begin{array}{c}
\hat{X}_{t}^{\leftarrow} \\
Y_{t}
\end{array}\right] \hat{X}_{t}^{\dagger}
$$

In this case our method of guaranteeing stability can be applied.

From the above it is seen that the estimate $\hat{A}$ can always be obtained in the form $\hat{A}=Z^{\leftarrow} Z^{\dagger}$ or $\hat{A}=Z^{\dagger} Z^{\uparrow}$ for some matrix $Z$. When $Z$ has finite dimensions the definitions of $Z^{\uparrow}$ and $Z^{\leftarrow}$ are ambiguous. In this paper we show that stability is guaranteed if $Z^{\uparrow}$ is obtained by introducing a block of zeros at the bottom, or if $Z^{\leftarrow}$ is obtained by introducing a block of zeros on the right. This result is quite surprising, since it is always enough to introduce only one block of zeros, irrespective of how many blocks there are altogether, and of the data they contain.

Previous proofs of stability in subspace methods, in so far as they exist at all, have relied on asymptotic arguments, as the number of data points is increased. But these do not hold for finite amounts of data, and it is not uncommon for unstable models to result. Vaccaro [9] suggested tapering the data in Kung-type algorithms, in order to obtain several properties, including stability. In [2] it is shown that stability can be guaranteed (and further properties obtained) in Kung's algorithm by extending the estimated impulse response (which forms the data in that case) by sufficiently many zeros.

\section{Guaranteeing stability}

Suppose that $Z \in \mathbf{R}^{N p \times n}$ is a matrix which is partitioned into blocks, each of size $p \times n$ :

$$
Z=\left[\begin{array}{l}
Z_{1} \\
Z_{2} \\
\vdots \\
Z_{N}
\end{array}\right]
$$

and let $Z_{o}^{\uparrow}$ denote the matrix which is formed when each block is shifted upwards by $p$ rows and a block of zeros is introduced at the bottom:

$$
Z_{o}^{\uparrow}=\left[\begin{array}{l}
Z_{2} \\
Z_{3} \\
\vdots \\
Z_{N} \\
0
\end{array}\right] .
$$

Also let $Z^{\dagger}$ denote the Moore-Penrose pseudo-inverse of $Z$, so that $Z^{\dagger} Z=I_{n}$. (We assume that $N p \geq n$.) 
Theorem 2.1 If

$$
\hat{A}=Z^{\dagger} Z_{o}^{\uparrow}
$$

then

$$
|\lambda(\hat{A})| \leq 1
$$

holds for any eigenvalue $\lambda(\hat{A})$ of $\hat{A}$.

Proof: Let $Z$ have the QR factorization [3]

$$
\begin{aligned}
Z & =Q R \\
& =\left[\begin{array}{l}
Q_{1} \\
Q_{2} \\
\vdots \\
Q_{N}
\end{array}\right] R
\end{aligned}
$$

where $Q^{T} Q=I$ and $R \in \mathbf{R}^{N p \times n}$ is upper-triangular. Then

$$
\begin{aligned}
Z_{o}^{\uparrow}= & Q_{o}^{\uparrow} R \\
= & {\left[\begin{array}{l}
Q_{2} \\
Q_{3} \\
\vdots \\
Q_{N} \\
0
\end{array}\right] R }
\end{aligned}
$$

and

$$
Z^{\dagger}=R^{\dagger} Q^{T}
$$

Now let $w$ be an eigenvector of $\hat{A}$, such that $\hat{A} w=\lambda w$. Then

$$
\begin{aligned}
\lambda R w & =R \hat{A} w \\
& =R\left(R^{\dagger} Q^{T} Q_{o}^{\uparrow} R\right) w .
\end{aligned}
$$

Let $\|$.$\| denote the Euclidean norm for vectors and the compatible (induced) norm for matrices.$ Then

$$
\begin{aligned}
|\lambda| \times\|R w\| & =\left\|R R^{\dagger} Q^{T} Q_{o}^{\uparrow} R w\right\| \\
& \leq\left\|R R^{\dagger}\right\| \times\left\|Q^{T} Q_{o}^{\uparrow}\right\| \times\|R w\| \\
& =\|R w\| .
\end{aligned}
$$

Hence $|\lambda| \leq 1$.

The last equality follows because

$$
R R^{\dagger}=\left[\begin{array}{ll}
I_{n} & 0 \\
0 & 0
\end{array}\right]
$$

and because of the following lemma:

Lemma 2.2 $\left\|Q^{T} Q_{o}^{\uparrow}\right\|=1$ 


$$
\begin{aligned}
\left(Q^{T} Q_{o}^{\uparrow}\right)^{T}\left(Q^{T} Q_{o}^{\uparrow}\right) & =\left(Q_{o}^{\uparrow}\right)^{T} Q_{o}^{\uparrow} \\
& =\sum_{k=2}^{N} Q_{k}^{T} Q_{k} \\
& =I-Q_{1}^{T} Q_{1} .
\end{aligned}
$$

Since the rows of $Q_{1}$ are orthonormal, $Q_{1}^{T} Q_{1}$ is an orthogonal projector [3], so all of its eigenvalues take the values 0 or 1 . Hence the eigenvalues of $I-Q_{1}^{T} Q_{1}$ take the values 1 or 0 . But the singular values of $Q^{T} Q_{o}^{\uparrow}$ are the nonnegative square roots of the eigenvalues of $\left(Q^{T} Q_{o}^{\uparrow}\right)^{T}\left(Q^{T} Q_{o}^{\uparrow}\right)$, and $\left\|Q^{T} Q_{o}^{\uparrow}\right\|$ is given by its largest singular value. Hence the lemma is proved.

Of course the following also holds, with

$$
\begin{aligned}
Z & =\left[Z_{1}, Z_{2}, \ldots, Z_{N}\right] \\
Z_{o}^{\leftarrow} & =\left[Z_{2}, \ldots, Z_{N}, 0\right] .
\end{aligned}
$$

\section{Theorem 2.3 If}

$$
\hat{A}=Z_{o}^{\leftarrow} Z^{\dagger}
$$

then

$$
|\lambda(\hat{A})| \leq 1
$$

holds for any eigenvalue $\lambda(\hat{A})$ of $\hat{A}$.

Proof: As for Theorem 2.1, taking transposes of everything.

The estimates of $A$ prescribed by Theorems 2.1 and 2.3 are just least-squares solutions of the equations $Z A=Z_{o}^{\uparrow}$ and $A Z=Z_{o}^{\leftarrow}$. In practice these equations are inconsistent (because the data in $Z$ is not generated by a linear system of the assumed order, and/or is corrupted by noise), and one could consider other solutions. In particular, a Total Least Squares (TLS) solution [3] has been suggested in [12]. It is interesting to note that our 'trick' does not guarantee stability if the TLS solution is taken.

\section{Conclusions}

Common practice seems to be to take

$$
Z=\left[\begin{array}{l}
Z_{1} \\
Z_{2} \\
\vdots \\
Z_{N-1}
\end{array}\right]
$$

instead of (14) and

$$
Z^{\uparrow}=\left[\begin{array}{l}
Z_{2} \\
Z_{3} \\
\vdots \\
Z_{N}
\end{array}\right]
$$


instead of (15), and indeed consistency of the resulting estimates can be demonstrated under certain assumptions [14]. Anecdotal evidence (see [12] for example) suggests that this gives more accurate results, for example in the sense of estimating the locations of lightly-damped poles more accurately. This is not surprising, since our procedure distorts the results by using a block of zeros in place of a block of data. Of course one expects this distortion to be very small if $N$ is large, and if the real system generating the data is stable.

It is quite easy to generate examples for which the use of (36) and (37) leads to unstable models, but it must be admitted that this is most likely to occur when the real system has poles close to the stability boundary, and this is precisely the case when our procedure is least likely to be useful. In particular, it is probably not suitable for applications such as high-resolution spectral analysis, because the bias introduced by it is likely to be excessive for those applications.

But we do not advocate that our procedure should be used routinely in all applications. It should be most useful in those applications in which some loss of accuracy is acceptable in return for guaranteed stability. This is likely to be the case in many applications in which subspace algorithms run on-line and unsupervised, such as adaptive control or fault monitoring. If they are used to provide the 'internal model' in a standard predictive control, or an 'internal model control' scheme, for example, then internal instability will result if the model produced is unstable, with much more disastrous results than would arise from some small bias on the model. It is also possible to use our method in parallel with a standard algorithm, using its results only if the standard algorithm yields an unstable model. Such an implementation would not destroy the consistency available with the standard algorithms (where this is achieved), because the probability of our modification being invoked will reduce to zero as the length of data increases.

\section{References}

[1] M.A.Budin, Minimal realization of discrete linear systems from input-output observations, IEEE Trans. Auto. Contr., AC-16, (1971), 395-401.

[2] C.T.Chou, Geometry of Linear Systems and Identification, PhD Thesis, Cambridge University, 1994.

[3] G.Golub, and C.Van Loan, Matrix Computations, (Johns Hopkins University Press, Baltimore, 1983).

[4] Gopinath,B, On the identification of linear time-invariant systems from input-output data, Bell System Tech. Jnl., 48, (1969), 1101-1113.

[5] B.L.Ho and R.E.Kalman, Effective construction of linear state-variable models from inputoutput functions, Regelungstechnik, 14, (1966), 545-548.

[6] S.Y.Kung, A new low-order approximation algorithm via singular value decomposition, Proc. 12th Asilomar Conf. on Circuits, Systems and Computers, (1978).

[7] W.E.Larimore, System identification, reduced-order filtering and modeling via canonical variate analysis, Proc.ACC, (1983), 445-451.

[8] M.Moonen, B.De Moor, L.Vandenberghe, J.Vandewalle, On and off-line identification of linear state-space models, Int. J. Contr., 49, (1989), 219-232. 
[9] R.J.Vaccaro, Finite-data algorithms for approximate stochastic realization, in: U.B.Desai, (ed), Modelling and Application of Stochastic Processes, (Kluwer, Boston 1986).

[10] A-J.Van Der Veen, E.F.Deprettere, and A.L.Swindlehurst, Subspace-based signal analysis using singular value decomposition, Proc. IEEE, 81, (1993), 1277-1308.

[11] P. Van Overschee, and B.DeMoor, N4SID: Subspace algorithms for the identification of combined deterministic stochastic systems, Automatica, 30, (1994), 75-93.

[12] P.Van Overschee, Subspace Identification: Theory, Implementation, Application, PhD thesis, Katholieke Universiteit Leuven, 1995.

[13] M.Verhaegen, and P.M.Dewilde, Subspace model identification, Parts 1 and 2, Int. J. Contr., 56, (1992), 1187-1241.

[14] M.Verhaegen, Identification of the deterministic part of MIMO state space models given in innovations form from input-output data, to appear in Automatica.

[15] J.C.Willems, From time series to linear systems, part II, Automatica, 22, (1986), 675-694.

[16] H.Zeiger and A.McEwen, Approximate linear realizations of given dimensions via Ho's algorithm, IEEE Trans. Auto. Contr., AC-19, (1974), 153. 